\title{
Modeling Dynamic Lift Response to Actuation
}

\author{
Xuanhong An* David R. Williams ${ }^{\dagger}$ \\ Illinois Institute of Technology, Chicago IL 60616, USA \\ Jeff D. Eldredge \\ University of California Los Angeles, Los Angeles CA 90095, USA \\ Tim Colonius ${ }^{\S}$ \\ California Institute of Technology, Pasadena CA 91125
}

\begin{abstract}
The dynamic lift response of an airfoil to sinusoidal amplitude variations from a synthetic jet actuator was studied. The wing was at a fixed angle of attack, and the actuator operated in a 'burst-mode' with a fixed duty cycle. The actuator burst amplitude was used as a control signal, which was varied between an 'off' condition and the actuator saturation voltage. Three dimensionless frequencies were examined, corresponding to $k=\frac{\pi f c}{U_{\infty}}=0.064,0.128$, and 0.25. Hysteresis loops in the lift increment were observed, whose shapes were dependent on the control frequency. Three different approaches to modeling the lift increment response were explored: a linear convolution approach, a nonlinear time delay and decay model, and a combination of those two. The linear convolution captures the high frequency content of the lift response, but becomes inaccurate when the actuator burst period is less than 3.5 convective times. The time delay and decay model reproduces the low frequency component of the lift response, but not the high frequency. When the control frequency becomes large, $(k=0.25)$, then the largest time-varying lift increment is produced near the minimum of the actuator voltage.
\end{abstract}

\section{Introduction}

$\mathrm{E}$ FFECTIVE flow control of the forces and moments acting on flight vehicles during transient flight condiEtions, such as, maneuvers or in turbulent flow conditions, can benefit greatly from having mathematical models of the system's response to actuator input. In the control theory community these are known as plant models, and they play a key role in the design of control systems. When written in the form of a transfer function, the plant model is the ratio of the output quantity (e.g., lift) to input quantity(e.g., actuator voltage). Feedforward controllers are often based on the inverse of the plant model, i.e., a desired lift is input to the inverse of the plant model to obtain the required voltage for the actuator.

'Dynamic' actuation in this paper refers to actuation that is changing in time, usually in response to external conditions. It is not simply the unsteadiness of the actuator itself. For example, the blades on wind turbines (Greenblatt, et al. ${ }^{1}$ ) and helicopter rotors experience periodic variations in the effective angle of attack at the frequency of blade rotation, and optimizing the performance of the blades with active flow control requires the actuators to change amplitude at the same frequency. Zero-net-mass-flow actuators (aka synthetic jets) are typically operated at much higher resonant frequencies, in order to maximize the jet exit velocity. The resonant frequency of the actuator is determined by its mechanical design, not the external flow that is to be controlled. It is not uncommon for there to be orders of magnitude difference between the actuator's operating frequency and the frequency associated with the fluid dynamic effects. To achieve dynamic flow control, the actuator must be operated at a lower frequency comparable to the characteristic frequency of the external flow field. In recent years, the so-called 'burst mode' of actuation has

\footnotetext{
*Research Assistant, MMAE Dept., Chicago IL 60616; xan2@hawk.iit.edu

†Professor, MMAE Dept., Chicago IL 60616, and Associate Fellow AIAA; david.williams@iit.edu

¥Professor, MAE Dept., Los Angeles CA 90095; eldredge@seas.ucla.edu, and Associate Fellow AIAA.

§Professor, Mech. and Civil Engr. Dept., Pasadena CA 91125; colonius@caltech.edu, and Associate Fellow AIAA
} 
been recognized as being a more effective method of controlling separated flows, than by either continuous excitation or individual pulses. Investigations by Wiltse and Glezer, ${ }^{2}$ Amitay and Glezer, ${ }^{3}$ and Margalit, ${ }^{4}$ determined that short duty cycle burst actuation is more effective than continuous actuation for increasing the lift coefficient. The 'burst mode' of actuation is used in this paper as a method to control the separated flow over an airfoil, and we explore different different approaches to modeling the lift response under time varying conditions.

The transient lift response to the onset and termination of actuation has been studied by several investigators. The transient response of the flow around a cylinder to pulsed amplitude modulation was examined by Amitay, et al. ${ }^{5}$ and Glezer \& Amitay ${ }^{6}$ showed the transient lift force response to step changes in actuation on a 2-D cylinder. The lift force was inferred from phase-averaed measurements of the circulation. They showed that the transient decay time for onset of actuation is much shorter (2-3 periods of vortex shedding) than the time following termination of actuation (15 periods of vortex shedding oscillation). The differences in these characteristic times is the reason behind the formation of the hysteresis loops shown in this paper. The details of transient forced reattachment and separation of flow over a generic 2D flap were obtained by Darabi \& Wygnanski. ${ }^{7,8}$ Stepwise onset of excitation showed a minimum reattachment time of 16 convective times $\left(t_{c o n v}=\frac{L_{\text {flap }}}{U_{\infty}}\right.$, which was dependent on flap angle, actuation frequency and amplitude. The stepwise termination of actuation required approximately 20 convective times for the fully separated flow to be re-established. The effect of a single cycle of a pulse-modulated actuator was used by Amitay \& Glezer ${ }^{9}$ to modify the lift on the separated flow around a 2D airfoil. The peak circulation was 2.3 times larger than the attached flow circulation. The characteristic period for the flow response was determined to be 30 actuator times to recover from a single-cycle (doublet-like) input signal to the actuator. It can be seen from the investigations of the separated flow around airfoils, that the time for lift enhancement at the onset of actuation is shorter than the time for lift recovery to the original separated flow state that occurs with the termination of actuation. The differences in these time scales is ultimately responsible for producing lift hysteresis when the actuation is periodic.

Previous work by our group Williams, et al. ${ }^{10}$ showed the ability of a linear convolution model to predict the dynamic lift response when a sequence of pulses from an actuator on a three-dimensional wing was applied to a separated flow. The kernel in the convolution integral was obtained from a single, short-duration pulse. The frequency and number of pulses was varied in the study, but the pulse amplitude was kept constant. The convolution model was able to capture the change in phase and amplitude as the pulse frequency was varied up to a certain duty cycle. In this paper we extend the convolution method to a 'burst mode' type of actuation that is operating at an optimized duty cycle. A sinusoidally varying amplitude is used to simulate a control signal. Different frequencies are used to examine the effect on lift control.

A second approach to modeling the lift response is also introduced in this paper, which is based on a nonlinear time delay and decay model. The model was motivated by the success of the Goman-Khrabrov ${ }^{11}$ model in capturing the lift hysteresis during pitching motions of an airfoil, Williams, et al. ${ }^{12}$ The time delay and decay modelling approach is shown to be effective in reproducing the important low frequency characteristics of the lift hysteresis. To include the high-frequency component of lift fluctuations, a hybrid model that combines the time delay and decay model with the convolution model is also examined.

The next section II explains the details of the burst actuation. The experimental methods are discussed in section III, and results are presented in section IV. The conclusions are given in section V.

\section{Burst actuation}

Zero net mass actuators have one or more resonant frequencies (Rathnasingham \& Breuer ${ }^{13}$ and Gallas ${ }^{14}$ ) that depend on the mechanical design details of the actuator. Because the largest jet exit velocities are obtained when the device is operated at one of its resonant frequencies, $f_{r}=\frac{1}{\Delta t_{p}}$, one would like to operate the actuator at that frequency. However, in most flow control situations the actuator resonant frequency $f_{r}$ has no connection with the characteristic frequencies of the external flow that one is trying to control. For example, in the experiment described in this paper the resonant frequency of the synthetic jet is $f_{r}=400 \mathrm{~Hz}$, while the period between bursts should be $T=3.5 t_{\text {conv }}$ ( or $f_{b}=3.5 \mathrm{~Hz}$ ) to produce the largest lift increment, where $t_{c o n v}=\frac{c}{U_{\infty}}$. Even lower frequencies are used for the flow control, which are approximately $f=1 \mathrm{~Hz}$ or less.

The diagram in Fig. 1 shows the three separate time scales and a voltage amplitude $A$ that are required to define a burst mode signal. The abscissa is time normalized by the convective time, so $t^{+}=\frac{t}{t_{\text {conv }}}$. The 
shortest time scale is $\Delta t_{p}$, which is the period of the actuator's resonant frequency. The burst time $\Delta t_{b}$ is defined by the number of sequential pulses $(n)$ from the actuator, so $\Delta t_{b}=n \Delta t_{p}$. For the results presented in this paper, $n=4$. The period between bursts is defined as $T$ is the longest time scale. The duty cycle of the burst is defined as $D T C=\frac{\Delta t_{b}}{T}$. The burst mode of actuation provides a method to reduce the actuator frequency to the desired optimal frequency for large lift increment. With a fixed duty cycle, the burst signal can be amplitude modulated at the frequency required for dynamic flow control.

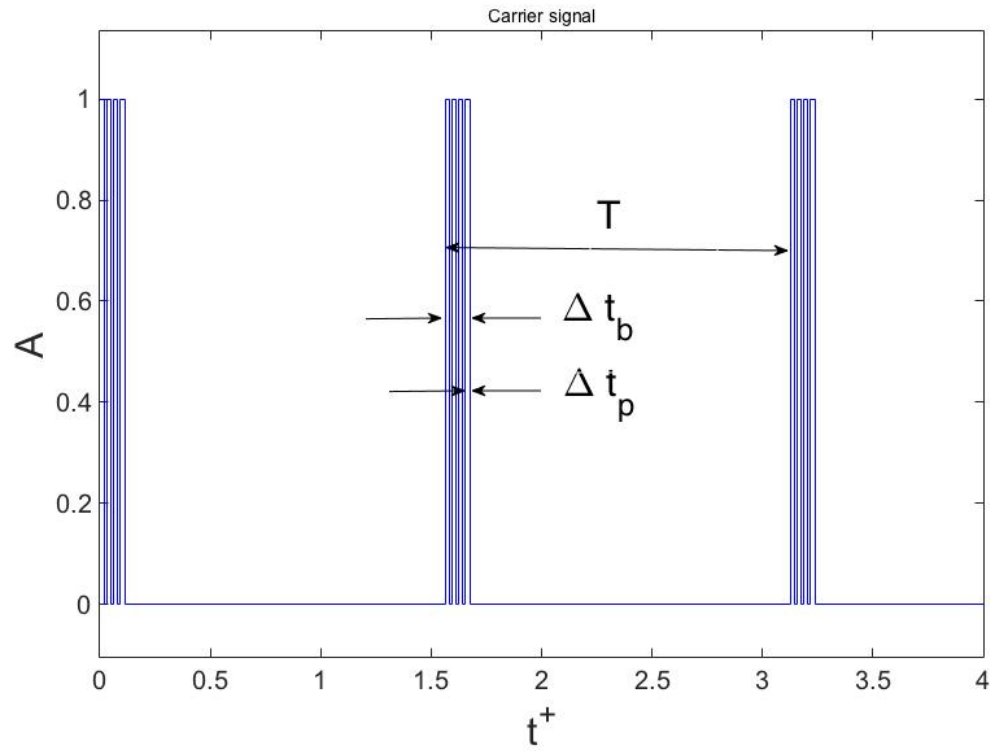

Figure 1. High frequency pulses in burst mode (carrier signal).

The low frequency flow control signal is shown in Fig. 2(a) is used to amplitude modulate the carrier signal. The combination of signals produces the actual voltage input signal to the actuator shown in Fig. 2(b).

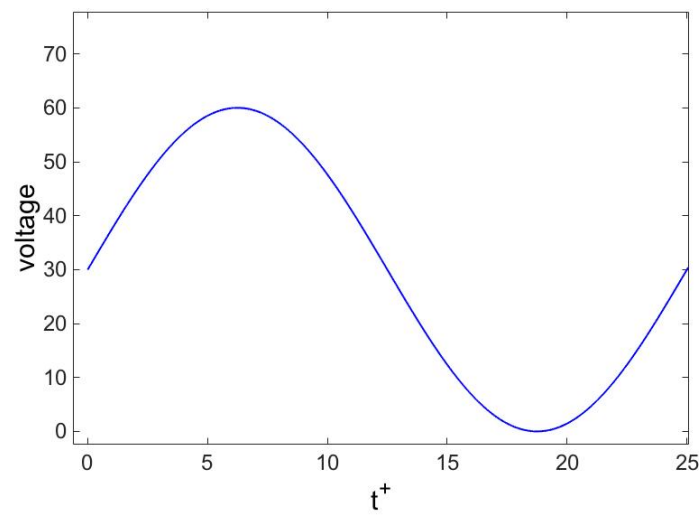

(a) Control Signal

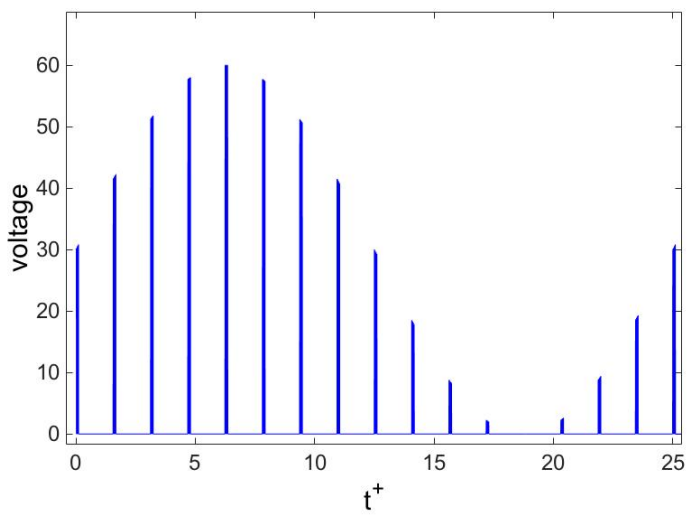

(b) Combined Voltage to Actuator

Figure 2. Components of burst-actuation. a) amplitude varying control signal; b) combination of signals sent to actuator 


\section{Experimental Approach}

The experiments were conducted in the Andrew Fejer Unsteady Flow Wind Tunnel at Illinois Institute of Technology. The test section cross-sectional dimensions are $0.6 \mathrm{~m} \mathrm{x} 0.6 \mathrm{~m}$. The freestream speed was $U_{\infty}=3 \mathrm{~m} / \mathrm{s}$ for all measurements presented here. A symmetric NACA 0009 airfoil with a chord $c=0.245 \mathrm{~m}$ was used as the test article. The chord-based Reynolds number was 49,000. The freestream turbulence was measured with a hot-wire anemometer and found to be 0.6 percent over the frequency band of $0.1 \mathrm{~Hz}$ to 30 Hz.. For the data presented in this paper, the angle of attack was fixed at either $\alpha=12^{\circ}$ or $\alpha=20^{\circ}$. The convective time $t_{c o n v}=\frac{c}{U_{\infty}}=0.0817 \mathrm{~s}$ was used as the characteristic time scale for normalization, and the dimensionless frequency was defined as $k=\frac{\pi f c}{U_{\infty}}$.

The airfoil was mounted to a 6-component ATI, Inc., Nano-17 force and moment transducer. Using phase-averaging techniques, the lift coefficient increment could be resolved to $\Delta C_{L}=0.01$.

The actuators were installed near the leading edge of the airfoil as shown in Fig. 3. The leading edge consisted of four segments, each with a pair of piezoelectric actuators. The jets from the actuators exited at the 7.3 percent chord location and a $30^{\circ}$ angle from the tangent to the airfoil surface. As discussed in section II the resonant frequency of each actuator was $400 \mathrm{~Hz}$. Hot wire measurements in the exit plane of the jets showed the peak exit velocities to be $4.9 \mathrm{~m} / \mathrm{s}$ when the actuator voltage was 60 Volts.

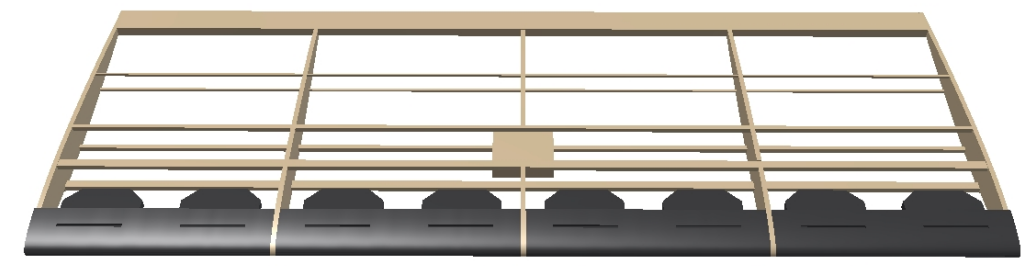

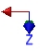

Figure 3. Drawing of NACA0009 airfoil with four pairs of actuators.

\section{Results}

When the actuator is operated continuously at a fixed duty cycle $\left(D T C=\frac{\Delta t_{b}}{T}=0.074\right)$ and constant burst amplitude of 60 Volts, the lift coefficient is increased above the non-actuated case as shown by the gray line in Fig. 4. The lift coefficient increment $\Delta C_{L}$ is the difference between the actuated and non-actuated lift, which can be non-zero when some degree of flow separation is present, $\alpha>10^{\circ}$. It is the interval between the actuated and non-actuated lift where dynamic active flow control can be employed.

The results in Fig. 4 correspond to steady state actuation. To understand how the dynamic lift behaves, we examine the lift response to single and multiple bursts from the actuator as shown in Fig. 5. This is similar to the approach used by Williams, et al. ${ }^{10}$ with a semi-circular wing. The airfoil angle of attack was fixed at $\alpha=20^{\circ}$, and 30 sets of data synchronized on the initial burst from the actuator were averaged to create the phase-averaged plots. The red line shows the voltage signal to the actuator, and the dark blue lines correspond to the experimental measurements. The cyan lines are the predicted lift response using the convolution integral approach, which is discussed in section A. In Fig. 5(a) the lift increment $\Delta C_{L}(t)$ following a single burst is plotted against time normalized by the convective time $t^{+}=\frac{t}{t_{\text {conv }}}$. An initial lift reversal occurs at $t^{+}=2$, which is followed by a rapid growth to the maximum lift increment at $t^{+}=3$. The initial lift reversal is indicative of the non-minimum phase behavior of the separated flow, as discussed by Kerstens, et al. ${ }^{15}$ The small amplitude, periodic oscillations during the lift increment decay (recovery of the flow to its initial separated state) correspond to vortex shedding from the airfoil, and are quite repeatable. The single burst lift response shown in this figure is used as the kernel in the convolution integral approach 


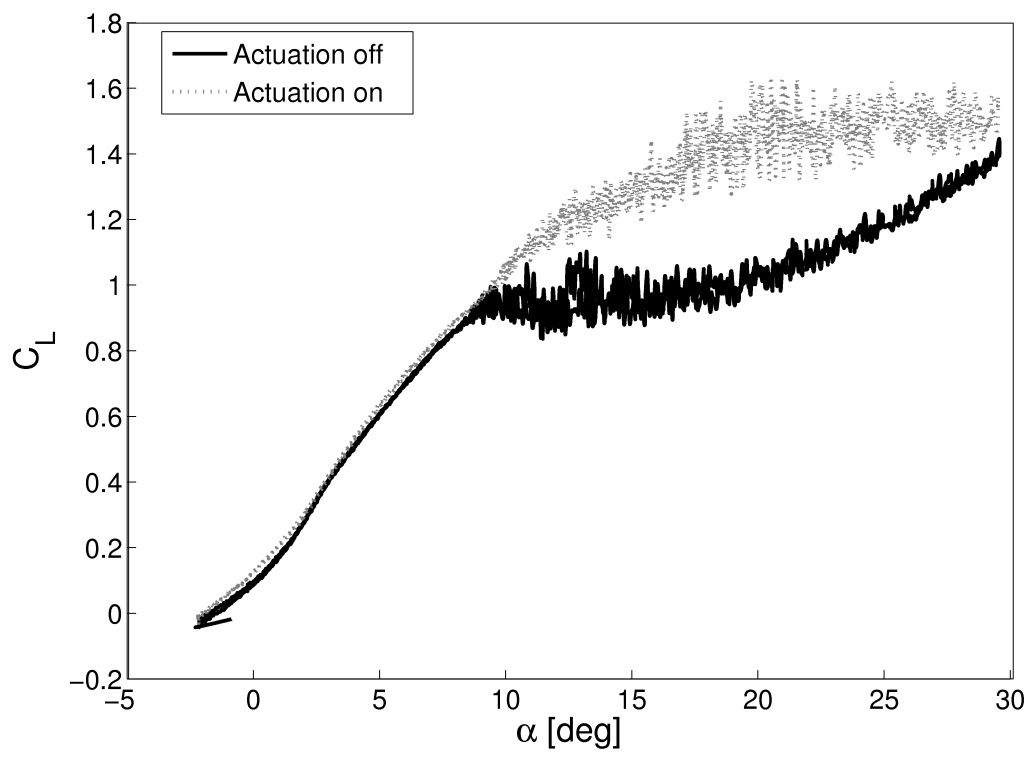

Figure 4. Steady state lift curve with actuation (gray line), without actuation (black line).

to predicting more complex lift behavior.

When a sequence of 10 bursts from the actuator with a period of $T=1.75 t_{\text {conv }}$ (or duty cycle $D T C=$ $0.07)$ is used, the time-varying lift increment shown in Fig. 5(b) is produced. The results show that it takes approximately three to four cycles before the lift increment reaches a 'steady state' conditiion. The oscillations in lift are synchronized with the bursts from the actuator. In Fig. 5(c) the period between bursts is increased to $T=3.5 t_{\text {conv }}$, which corresponds to a reduced duty cycle $D T C=0.035$. This duty cycle produces the largest lift increment. Current speculation about the reason for the differences in lift increment between the two duty cycles is that nonlinear interactions between the vortex structures produced by the actuator bursts are interfering with each other in the longer duty cycle case, but reinforcing each other in the case of $D T C=0.035$. PIV measurements are currently underway to determine if the speculation is correct or not.

In Fig. $5(\mathrm{~d})$ the period between actuator bursts was increased to $T=7 t_{c o n v}$, which reduces the duty cycle to $D T C=0.0175$. This is also a non-optimal case, which has a reduced lift increment compared to Fig. 5(c). In this case we speculate that each burst is 'more independent', i.e., less interaction between vortices produced by each burst occurs, and again the maximum lift increment is reduced relative to the optimal burst period.

\section{A. Convolution model}

The convolution approach is based on the idea that a single burst from the actuator is short enough in time to approximate an impulse disturbance to the separated flow 'system', and the corresponding lift increment $\left(\Delta C_{L}\right.$ single $)$ approximates an impulse response function. The convolution of the impulse response function with the discrete voltage signal $\mathrm{V}(\mathrm{k}-\mathrm{j})$ is given by Eq. 1 .

$$
\Delta C_{L}(k)=\sum \Delta C_{L} \text { single }(j) V(k-j)
$$

The convolution model is tested with a sequence of 10 bursts. Each burst consists of 4 pulses, so $\Delta t_{b}=0.01 \mathrm{~s}=0.21 t_{\text {conv }}$. The period between bursts is varied between $1.75 t_{\text {conv }} \leq T \leq 7.0 t_{\text {conv }}$ The predictions of the lift increments by the convolution approach are shown by the cyan lines in Figs. 5(a-d). The agreement between the model and experiment is good for the smaller duty cycle cases, when the interval between bursts is greater than $3.5 t_{\text {conv }}$, but this linear modeling approach does not work when the bursts 


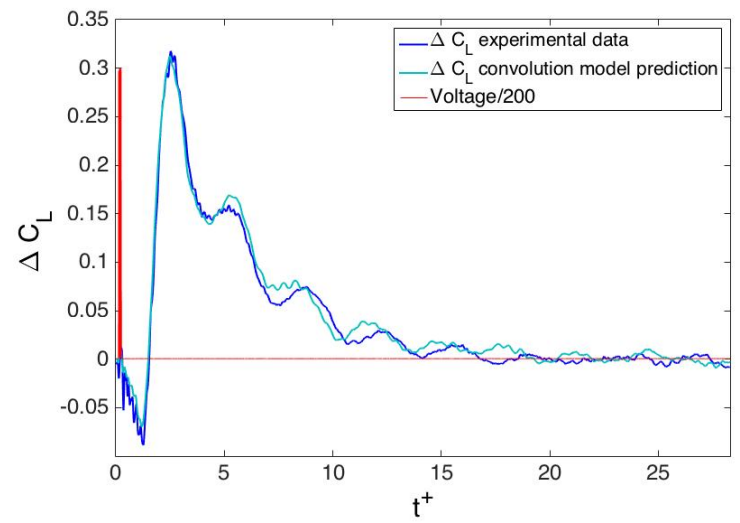

(a) Single burst, $\mathrm{T} \rightarrow \infty$

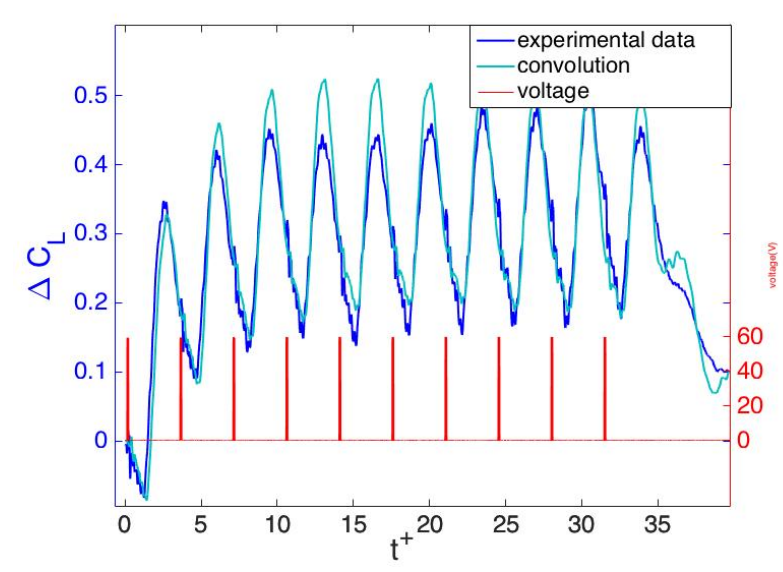

(c) 10 bursts, $T=3.5 t_{\text {conv }}$

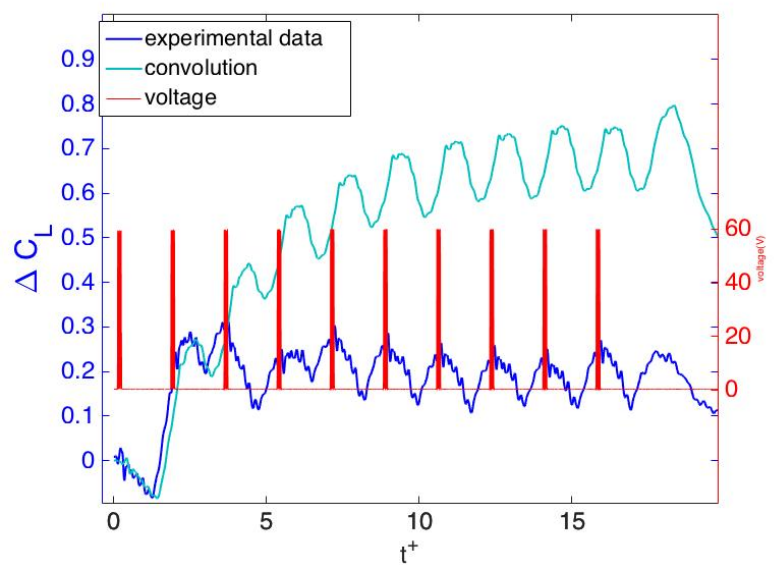

(b) 10 bursts, $T=1.75 t_{\text {conv }}$

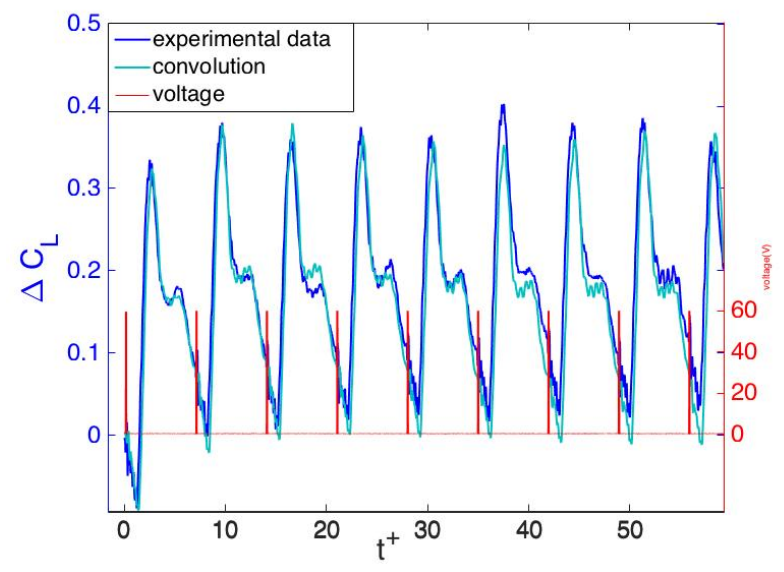

(d) 10 bursts, $T=7 t_{\text {conv }}$

Figure 5. Lift response to burst-actuation at $\alpha=20^{\circ}$. The red lines show the voltage to the actuators. a) single burst at $\mathbf{t}=0 ; \mathbf{b}) 10$ repeated bursts at the optimal $\left.T=1.75 t_{\text {conv }} ; \mathbf{c}\right) \mathbf{1 0}$ repeating bursts at $\left.T=3.5 t_{\text {conv }} ; \mathbf{d}\right) \mathbf{1 0}$ repeating bursts at $T=7 t_{\text {conv }}$. 
are closer together. Again, interactions between the vorticity fields produced by the individual bursts are expected to be nonlinear, so a linear model may not be valid in this regime.

\section{B. Time delay and decay model}

A different approach to modeling the dynamic hysteresis behavior of the lift response to actuation was developed by the first author. The approach is motivated by the success of the nonlinear time delay model for dynamic lift response of aircraft introduced by Goman \& Khrabrov. ${ }^{11}$ The time delay and decay (TDD) model consists of a first order equation (eqn. 2) in $x$, where $x$ represents the instantaneous lift increment normalized by the maximum achievable lift increment, i.e., $x=\frac{\Delta C_{L}}{\Delta C_{L} \max }$. The model contains two time constants $\left(\tau_{1}\right.$ and $\left.\tau_{2}\right)$ and a nonlinear forcing function $x_{0}(V)$. The function $x_{0}(\alpha)$ is measured directly from lift increment data obtained at steady state conditions, and is shown in Fig. 6 . The values of the time constants were found by trial and error fitting to one dynamic control case, and the values were determined to be $\tau_{1}=2.5 t_{\text {conv }}$, and $\tau_{2}=2.0 t_{\text {conv }}$. The first time constant $\tau_{1}$ is representative of the recovery of the flow to a separated state following the termination of actuation. The second time constant $\tau_{2}$ is more difficult to connect with the flow physics, but partially represents a time delay between the onset of actuation and the lift response.

$$
\tau_{1} \frac{d x}{d t}+x=x_{0}\left[V\left(t-\tau_{2}\right)\right]
$$

where

$$
\Delta C_{L}=x \Delta C_{L} \max
$$

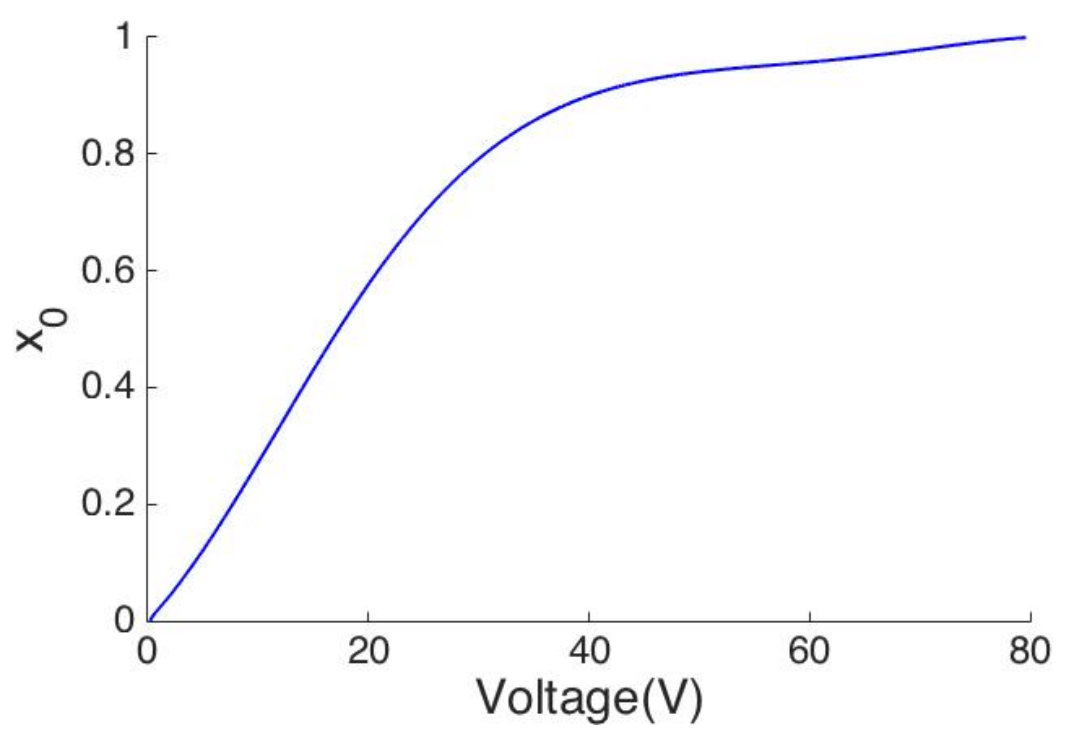

Figure 6. Nonlinear forcing function for Eq. 2

The delay and decay model (TDD) predictions of the lift response are shown in Fig. 7 as the red lines. The experimental measurements are shown by the blue lines. The burst period was shortened to $T=1.56 t_{c o n v}$ to provide better time resolution during each cycle of the control. A tradeoff of reduced lift increment occurs for the shorter burst spacing.

Clearly the TDD model has no ability to capture the high frequency lift oscillations associated with the bursts from the actuator, but after a short transient following the start of the numerical integration the TDD solution stabilizes onto a closed trajectory that reproduces the low frequency hysteresis observed in the dynamic lift increment data. The trajectory is counter-clockwise around the loops. An interesting effect occurs as the control frequency is increased from $f=0.25 \mathrm{~Hz}$ Fig. 7a to $f=1.0 \mathrm{~Hz}$ Fig. 7c. At the low 
frequency the lift increment is maximum at the maximum actuator voltage, but as the frequency increases, the maximum lift increment occurs near 0 Volts actuator input!

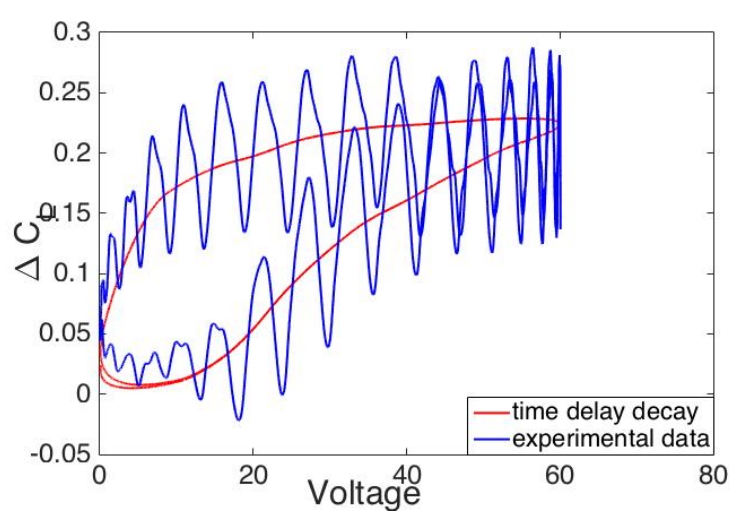

(a) $k=0.064, f=0.25 \mathrm{~Hz}$

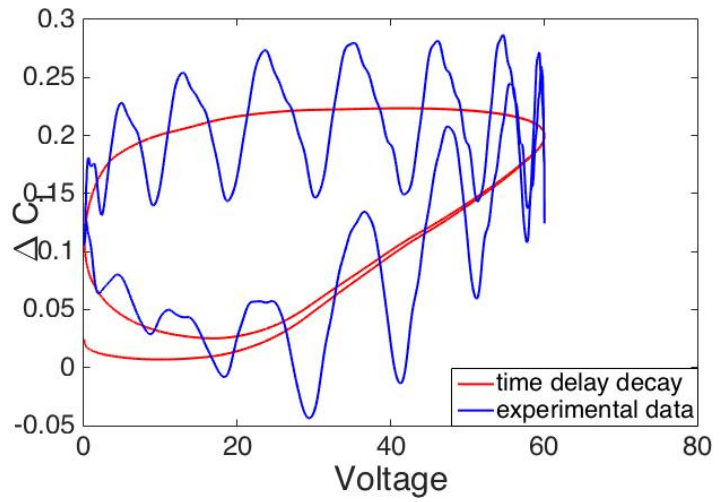

(b) $k=0.128, f=0.5 H z$

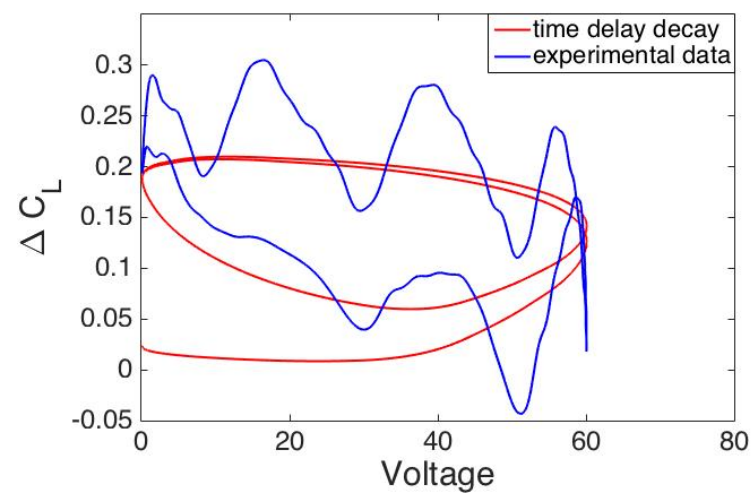

(c) $k=0.25, f=1.0 H z$

Figure 7. Nonlinear delay and decay model predictions of the lift response to sinusoidally varying amplitude of burst-actuation for $\alpha=12^{\circ}$. The blue lines show the measured lift increment and the red lines show the nonlinear model prediction. a) $k=0.064$; b) $k=0.128$; c) $k=0.25$.

The role of the time constants, $\tau_{1}$ and $\tau_{2}$, and the dependence of the shape of the hysteresis loop, including the phenomenon of the maximum lift increment occurring near the minimum voltage, can be partially explained by examining a linearized version of the TDD model. To linearize we assume there are small amplitude variations of the voltage about a stationary point in the $X_{0}$ vs. voltage plot, so that $V\left(t-\tau_{2}\right)=V_{s}+u\left(t-\tau_{2}\right)$ and $X_{0}=X_{s}+x(t)$. Substituting into equation 2 and subtracting the steady state equation gives

$$
\tau_{1} \frac{d x}{d t}+x=x_{0}\left[V_{s}+u\left(t-\tau_{2}\right)\right]-X_{0}\left[V_{s}\right]
$$

The nonlinear forcing term on the right can be approximated as

$$
x_{0}\left[V_{s}+u\left(t-\tau_{2}\right)\right] \approx X_{0}\left[V_{s}\right]+\left.\frac{d X_{0}}{d V}\right|_{V_{s}} u\left(t-\tau_{2}\right) .
$$

Taking the Laplace transform of the equation 5, and using a first order approximation for the time delay term gives

$$
\tau_{1} s X(s)+X(s) \approx \kappa U(s) \frac{1}{\tau_{2} s+1},
$$

where $\kappa=\left.\frac{d X_{0}}{d V}\right|_{s}$. 
The transfer function can be written

$$
\frac{X(s)}{U(s)}=\kappa \frac{1}{\left(\tau_{1} s+1\right)\left(\tau_{2} s+1\right)} .
$$

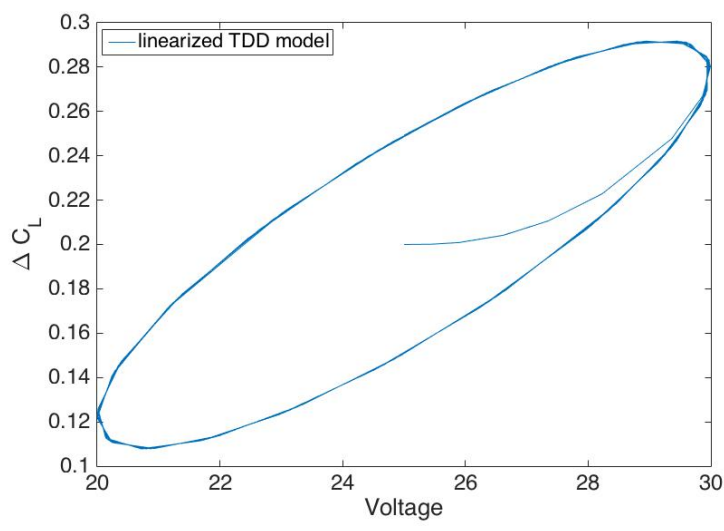

(a) $k=0.064, f=0.25 H z$

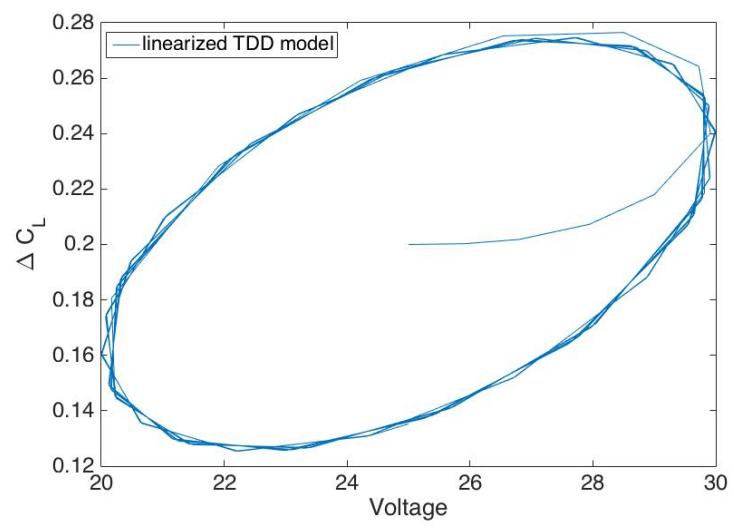

(b) $k=0.128, f=0.5 H z$

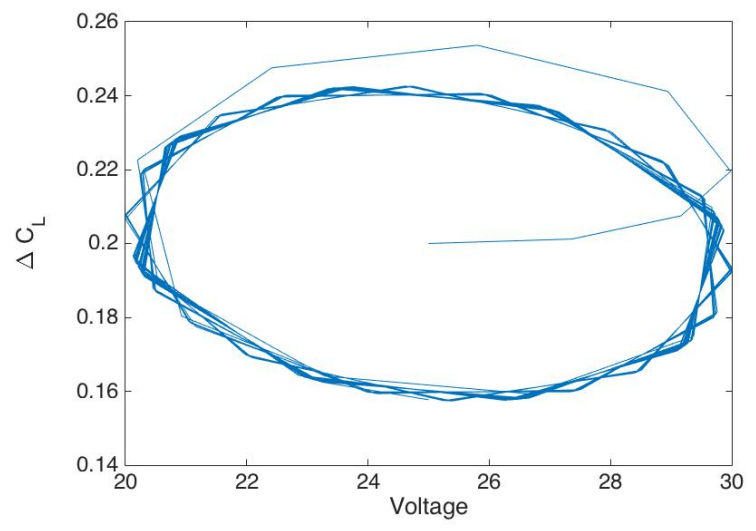

(c) $k=0.25, f=1.0 H z$

Figure 8. Linearized delay and decay model predictions of the lift response to sinusoidally varying $5 \mathrm{~V}$ amplitude of actuation. The blue lines show the lift increments predicted by equation 7 . a) $k=0.064 ; \mathbf{b}) k=0.128 ; \mathbf{c}) k=0.25$.

The linear model and figures 8 a-c show that the delay and decay time constants are responsible for the general hysteresis loop formation. We also see that as the frequency increases, the loops 'rotate' so that a maximum lift increment is achieved near the minimum voltage point. The linear model produces an elliptic shaped hysteresis loops, while the large amplitude nonlinear model generates a distorted ellipse (paisley-shaped) loop. This can be attributed to the nonlinearity of the $X_{0}$ function.

\section{Hybrid convolution TDD model}

A third approach to modeling the dynamic lift increment was obtained by combining the convolution and TDD methods. We recognize that this is a completely ad hoc approach, but we found the results to be interesting. The convolution integral (Eq. 1) is used to obtain an estimate of the dynamic lift increment with a sinusoidally varying actuator amplitude. Because the burst period is too short for the linear approach to be accurate, the convolution over-predicts the amplitude of the low frequency component, as shown by the blue line in Fig. 9. But the high frequency oscillations associated with the bursts are captured and their amplitudes are nearly correct. Next the signal is high-pass filtered to remove the low frequency component as shown by the red line in Fig. 9.

The high pass filtered signal is then superposed with the low frequency component from the TDD model discussed in section B. The results of the combined model are shown in Fig. 10 for three different forcing 


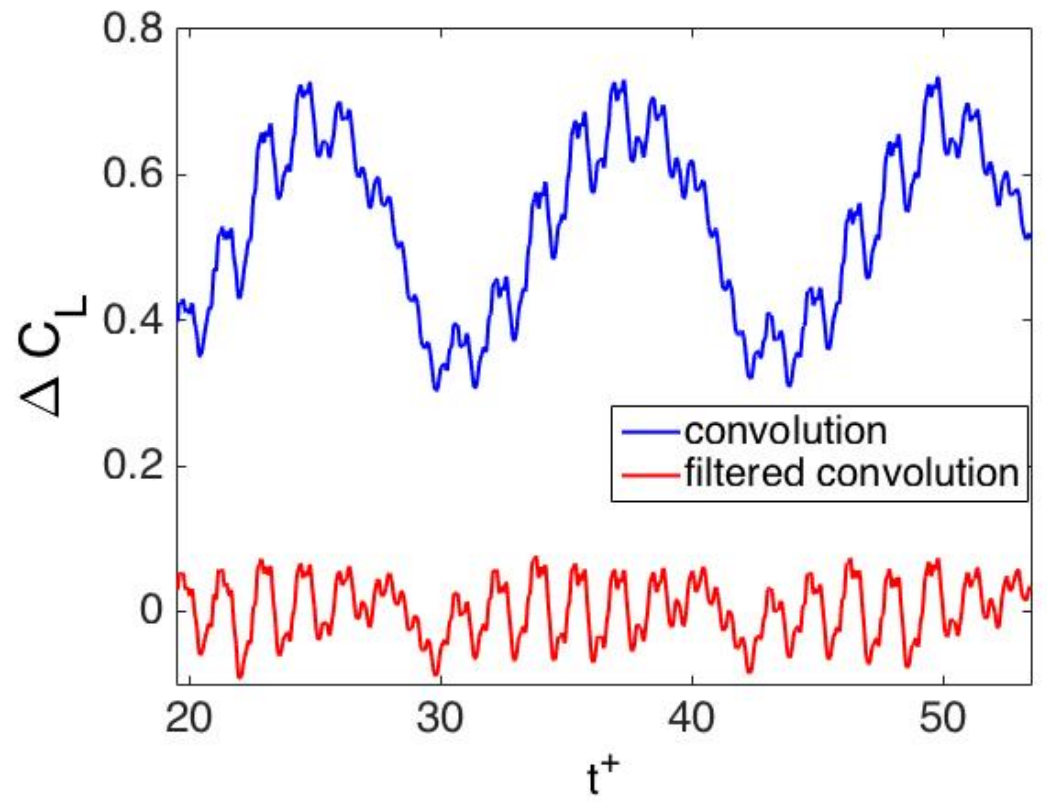

Figure 9. Standard convolution integral of the sinusoidally varying burst amplitude signal (blue line), and after high-pass filtering (red line).

frequencies, $k=0.064,0.128$, and $k=0.25$. The experimental data is shown by the blue lines, which is compared to the combined model (red lines) and the modified convolution model (black lines). The combined model captures the high frequency lift component better than the modified convolution approach.

\section{Conclusion}

Three methods of modeling the dynamic lift increment were examined using an NACA 0009 airfoil with flow control actuators that are operated with open-loop sinusoidal forcing. The 'burst mode' of actuation is used to obtain large lift increments, and the amplitude of the burst signal is modulated to obtain a timevarying (dynamic) lift coefficient. The resulting lift increments form hysteresis loops when the lift increment is plotted against the actuator voltage. The burst components from the actuators create large amplitude variations in the lift increment at higher frequencies than the low frequency sinusoidal control signal. A linear convolution integral approach, a nonlinear time delay and decay model, and a combination of the two methods were used to reproduce the measured lift increment data. The nonlinear time delay and decay model reproduces the low-frequency components of hysteresis, but is not capable of capturing the high-frequency burst component in the lift signal. The convolution approach reproduces both the hysteresis and the high frequency burst components, but it over predicts the amplitude of the lift signal when the burst period is shorter than $3.5 t_{c o n v}$. When the sinusoidal control frequency was $1 \mathrm{~Hz}(k=0.25)$, the lift increment reached its maximum when the actuator voltage was near its minimum value. A linearized version of the TDD model showed that this behavior was the result of the delay and decay time constants. A hybrid model obtained by combining the high frequency component from the convolution model with the low frequency lift from the time delay and decay was the closest to the experimental measurements.

\section{Acknowledgments}

Support by the U.S. Air Force Office of Scientific Research (FA9550-14-1-0328) with program manager Dr. Douglas Smith is gratefully acknowledged. 


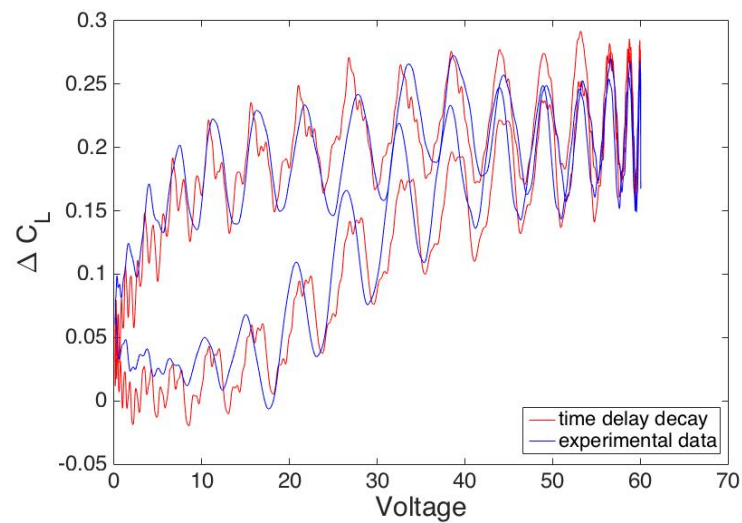

(a) $k=0.064, f=0.25 H z$

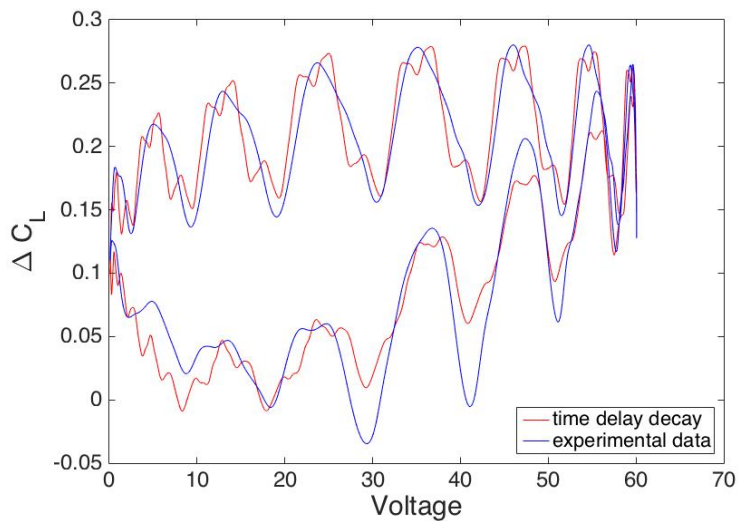

(b) $k=0.128, f=0.5 \mathrm{~Hz}$

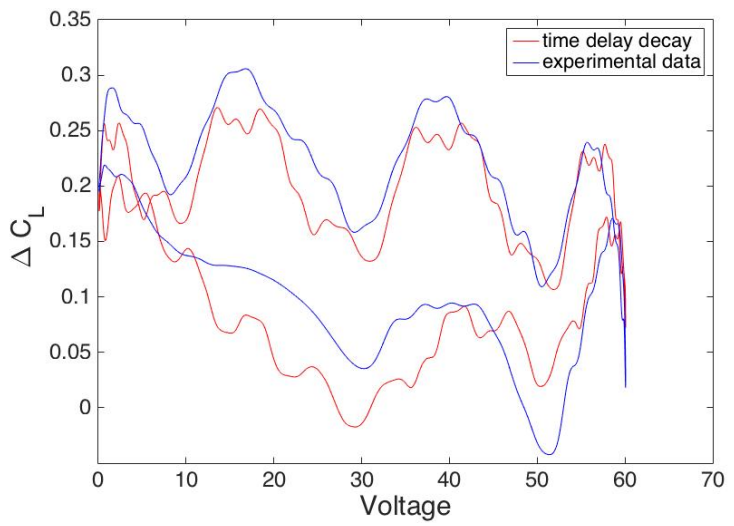

(c) $k=0.25, f=1.0 H z$

Figure 10. Combined convolution and time delay and decay model predictions of the lift response to sinusoidally varying $60 \mathrm{~V}$ amplitude of actuation. The blue lines show the measured lift increment and the red lines show the combined model prediction. a) $k=0.064$; b) $k=0.128 ; \mathbf{c}) k=0.25$. 


\section{References}

${ }^{1}$ Greenblatt, D., Mueller-Vahl, H., Lautman, R., Ben-Harav, A., and Eshel, B., "DBD Plasma-Based Flow Control on a Vertical Axis Wind Turbine," Active Flow Control III - R. King, ed., Vol. 127, 2014, pp. 71-86.

${ }^{2}$ Wiltse, J. M. and Glezer, A., "Manipulation of Free Shear Flows Using Piezoelectric Actuators," J Fluid Mech, Vol. 249, 1993, pp. 261.

${ }^{3}$ Amitay, M. and Glezer, A., "Controlled transients of flow reattachment over stalled airfoils," Intl. J. Heat and Fluid Flow, Vol. 23, 2002, pp. 690-699.

${ }^{4}$ Margalit, S., Greenblatt, D., Seifert, A., and Wygnanski, I., "Delta Wing Stall and Roll Control Using Segmented Piezoelectric Fluidic Actuators," Journal of Aircraft, Vol. 42, No. 3, 2005, pp. 698-709.

${ }^{5}$ Amitay, M., Simth, B. L., and Glezer, A., "Aerodynamic Flow Control using Synthetic Jet Technology," AIAA Paper 98-0208, AIAA, 1998.

${ }^{6}$ Glezer, A. and Amitay, M., "Synthetic Jets," Annu. Rev. Fluid Mech., Vol. 34, 2002, pp. 503-529.

${ }^{7}$ Darabi, A. and Wygnanski, I., "Active management of naturally separated flow over a solid surface. Part 1 . The forced reattachment process," Journal of Fluid Mechanics, Vol. 510, No. 1, 2004, pp. 105-129.

${ }^{8}$ Darabi, A. and Wygnanski, I., "Active management of naturally separated flow over a solid surface. Part 2. The separation process," Journal of Fluid Mechanics, Vol. 510, No. 1, 2004, pp. 131-144.

${ }^{9}$ Amitay, M. and Glezer, A., "Flow transients induced on a $2 \mathrm{D}$ airfoil by pulse modulated actuation," Experiments in Fluids, Vol. 40, 2006, pp. 329-333.

${ }^{10}$ Williams, D., Tadmor, G., Colonius, T., Kerstens, W., Quach, V., and Buntain, S., "Lift response of a stalled wing to pulsatile disturbances," AIAA Journal, Vol. 47, No. 12, 2009, pp. 3031-3037.

${ }^{11}$ Goman, M. and Khrabrov, A., "State-Space Representation of Aerodynamic Characteristics of an Aircraft at High Angles of Attack," Journal of Aircraft, Vol. 31, No. 5, 1994, pp. 1109-1115.

${ }^{12}$ Williams, D., X., A., S., I., King, R., and Reißner, F., "Dynamic Hysteresis Control of Lift on a Pitching Wing," Experiments in Fluids, Vol. 56, No. 5, 2015.

${ }^{13}$ Rathnasingham, R. and Breuer, K. S., "Coupled fluid-structural characteristics of actuators for flow control," AIAA Journal, Vol. 35, 1997, pp. 832-837.

${ }^{14}$ Gallas, Q., Homan, R., Nishida, T., Carroll, B., Sheplak, M., and Cattafesta, L., "Lumped element modeling of piezoelectric-driven synthetic jet actuators," AIAA Journal, Vol. 41, 2003, pp. 240-247.

${ }^{15}$ Kerstens, W., Williams, D., Pfeiffer, J., King, R., and Colonius, T., "Closed-Loop Control of Lift for Longitudinal Gust Suppression at Low Reynolds Numbers," AIAA Journal, Vol. 49, No. 8, 2011, pp. 1721-1728. 